\title{
SPECTRAL PROPERTIES AND DISTRIBUTION OF EIGENVALUES OF NON-SELF-ADJOINT ELLIPTIC DIFFERENTIAL OPERATORS
}

\author{
Reza Alizadeh $^{1 \S}$, Ali Sameripour ${ }^{2}$ \\ 1,2 Mathematics Dept., Lorestan University \\ Khoramabad, IRAN
}

\begin{abstract}
Let $\Omega$ be a bounded domain in $R^{n}$ with smooth boundary $\partial \Omega$. In this article, we investigate the spectral properties of a non-selfadjoint elliptic differential operator $(P u)(x)=-\sum_{i, j=1}^{n}\left(\omega^{2 \alpha}(x) a_{i j}(x) Q(x) u_{x_{i}}^{\prime}(x)\right)_{x_{j}}^{\prime}$, acting in the Hilbert space $H_{\ell}=L^{2}(\Omega)^{\ell}$ with Dirichlet-type boundary conditions. Here $c|s|^{2} \leq \sum_{i, j=1}^{n} a_{i j}(x) s_{i} \overline{s_{j}} \quad\left(s=\left(s_{1}, \ldots, s_{n}\right) \in \mathbf{C}^{n}, \quad x \in \Omega\right), a_{i j}(x)=$ $\overline{a_{j i}(x)} \in C^{2}(\bar{\Omega}), \quad 0 \leq \alpha<1$. Furthermore, suppose that $\omega \in C^{1}(\bar{\Omega}, \mathcal{R})$ and this function is a positive function and called the weight function and $Q(x) \in C^{2}\left(\bar{\Omega}\right.$, End $\left.\mathbf{C}^{\ell}\right)$ such that for each $x \in \bar{\Omega}$ the matrix function $Q(x)$ has non-zero simple eigenvalues $\mu_{j}(x) \in C^{2}(\bar{\Omega}, \mathbf{C}) \quad(1 \leq j \leq \ell)$ lie in the $\psi_{\theta_{1} \theta_{2}}$ and real numbers and here $\psi_{\theta_{1} \theta_{2}}=\left\{z \in \mathbf{C}: \pi / 2<\theta_{1} \leq|\arg z| \leq \theta_{2}<\pi\right\}$,
\end{abstract}

AMS Subject Classification: 47F05, 35JXX, 35PXX

Key Words: resolvent; asymptotic spectrum; eigenvalues; non-selfadjoint elliptic differential operators

\section{Introduction}

Let $\Omega$ be a bounded domain in $R^{n}$ with smooth boundary $\partial \Omega$ (i.e., $\partial \Omega \in C^{\infty}$ ). We introduce the weighted Sobolev space $\mathcal{H}=W_{2, \alpha}^{2}(\Omega)$ corresponding to the non-selfadjoint elliptic differential operator

Received: December 4, 2020

(C) 2021 Academic Publications

${ }^{\S}$ Correspondence author 


$$
(P u)(x)=-\sum_{i, j=1}^{n}\left(\omega^{2 \alpha}(x) a_{i j}(x) Q(x) u_{x_{i}}^{\prime}(x)\right)^{\prime}{ }_{x_{j}},
$$

acting in the Hilbert space $H_{\ell}=L^{2}(\Omega)^{\ell}$. The weighted Sobolev space $\mathcal{H}=$ $W_{2, \alpha}^{2}(\Omega)$ is the space of complex value functions $u(x)$ defined on $\Omega$ with the finite norm:

$$
|u|_{+}=\left(\sum_{i=1}^{n} \int_{\Omega} \omega^{2 \alpha}(x)\left|u_{x_{i}}^{\prime}(x)\right|^{2} d x+\int_{\Omega}|u(x)|^{2} d x\right)^{1 / 2} .
$$

We denote by $\stackrel{\circ}{\mathcal{H}}$ the closure of $C_{0}^{\infty}(\Omega)$ in $\mathcal{H}$ with respect to the above norm. That is, $\mathcal{H}$ is the closure of $C_{0}^{\infty}(\Omega)$ in $W_{2, \alpha}^{2}(\Omega)$. The notion $C_{0}^{\infty}(\Omega)$ stands for the space of infinitely differentiable functions with compact support in $\Omega$. In this paper, we investigate the spectral properties, in particular we estimate the resolvent of a non-selfadjoint elliptic differential operator of type

$$
(P u)(x)=-\sum_{i, j=1}^{n}\left(\omega^{2 \alpha}(x) a_{i j}(x) Q(x) u_{x_{i}}^{\prime}(x)\right)_{x_{j}}^{\prime}
$$

acting in Hilbert space $H_{\ell}=L^{2}(\Omega)^{\ell}$ with Dirichlet-type boundary conditions. Here $0 \leq \alpha<1, \quad a_{i j}(x)=\overline{a_{j i}(x)}(i, j=1, \ldots, n), \quad a_{i j}(x) \in C^{2}(\bar{\Omega}) \quad(i, j=$ $1, \ldots, n)$, and the functions $a_{i j}(x)$ satisfy the uniformly elliptic condition, i.e., there exists $c>0$ such that:

$$
c|s|^{2} \leq \sum_{i, j=1}^{n} a_{i j}(x) s_{i} \overline{s_{j}} \quad\left(s=\left(s_{1}, \ldots, s_{n}\right) \in \mathbf{C}^{n}, x \in \Omega\right) .
$$

Furthermore, suppose that $Q(x) \in C^{2}\left(\bar{\Omega}\right.$, End $\left.\mathbf{C}^{\ell}\right)$ such that for each $x \in \bar{\Omega}$, the matrix function $Q(x)$ has non-zero simple eigenvalues $\mu_{j}(x) \in C^{2}(\bar{\Omega}) \quad(1 \leq j \leq \ell)$ lie in the $\psi_{\theta_{1} \theta_{2}}$, and real numbers. Here $\psi_{\theta_{1} \theta_{2}}=\left\{z \in \mathbf{C}: \pi / 2<\theta_{1} \leq|\arg z| \leq\right.$ $\left.\theta_{2}<\pi\right\}, \mu_{1}(x), \ldots, \mu_{\nu}(x)$ are real and the rest of them, i.e.

$$
\mu_{\nu+1}(x), \ldots, \mu_{\ell}(x) \in \psi_{\theta_{1} \theta_{2}} .
$$

We closed extension the operator $P$ with respect to the weighted Sobolev space $\mathcal{H}=W_{2, \alpha}^{2}(\Omega)$, then here we extend the domain of operator $P$ from the domain in the Hilbert space $H_{\ell}=L^{2}(\Omega)^{\ell}$ to the big and closed domain of the weighted Sobolev space $\mathcal{H}^{\ell}=W_{2, \alpha}^{2}(\Omega)^{\ell}$. Therefore,

$$
D(P)=\left\{v \in(\stackrel{\circ}{\mathcal{H}})^{\ell} \cap\left(W_{2, \mathrm{loc}}^{2}(\Omega)\right)^{\ell}: P v \in H_{\ell}\right\}
$$

(see [8]), where the local space $W_{2, l o c}^{2}(\Omega)$ is the functions $u(x)$ $(x \in \Omega)$ of the form 


$$
W_{2, l o c}^{2}(\Omega)=\left\{u \in L^{2}(\Omega): \sum_{i=0}^{2} \int_{J}\left|u^{(i)}(x)\right|^{2} d x<\infty\right\},
$$

where $J$ is an arbitrary open subset of $\Omega$.

Before entering into the main discussion of the article, it is reminded that the Sturm-Liouville eigenvalue problem is as

$$
-\left(p(x) y^{\prime}\right)^{\prime}+q(x) y=\lambda \omega(x) y: x \in[a, b] .
$$

In this equation $p, q, \omega$ are real valued functions and $p, \omega>0, p(x), p^{\prime}(x)$, $\omega(x), q(x) \in C[a, b]$. The spectral properties of this operator have been discussed in many articles. What has been done in this paper is that the $q$ and $p$ are considered as complex-valued functions. That makes the linear operator non-self-adjoint. Since there is no general spectral theory for non-self-adjoint operators, it becomes difficult to study the spectrum of this operator. By considering the Sobolev space and defining a new norm in this space, we try to obtain an estimate of the resolvent of this operator. In this context, we refer the reader to the effective studies in the works [2] and [10] by Boimatov et al. and Sameripur et al.

\section{Preliminaries}

Let $H$ be a separable Hilbert space and $T$ be a densely defined linear operator with $D(T) \subset H$.

Definition 1. The set of complex numbers

$$
W(T):=\{<T u, u>\in C: u \in D(T),\|u\|=1\}
$$

is called the numerical range of $T$.

Definition 2. The operator $T$ is called sectorial with semi-angle $\theta \in\left[0, \frac{\pi}{2}\right)$ and vertex at $z=0$ if

$$
W(T) \subseteq \Phi(\theta):=\{z \in C:|\arg z| \leq \theta\} .
$$

If in addition $T$ is closed and there is $z \in C \backslash \Phi(\theta)$ such that it is belong to the resolvent set $\rho(T)$, then operator $T$ is called $m$-sectorial. 
Definition 3. An eigenvalue of a linear operator $T$ in Hilbert space $H$ is a complex number $\lambda$ that there exists non zero vector $u$ in $H$ such that $T u=\lambda u$. We say $u$ is an eigenvector of $T$ corresponding to $\lambda$.

\section{The resolvent estimate of degenerate elliptic differential operators on $H$}

Theorem 4. Assume that the operator

$$
P u=-\sum_{i, j=1}^{n}\left(\omega^{2 \alpha}(x) a_{i j}(x) q(x) u^{\prime}\left(x_{i}\right)\right)^{\prime} x_{j}
$$

acts in the Hilbert space $H=L^{2}(\Omega)$ with Dirichlet-type boundary conditions, and let us consider the sector

$$
\psi_{\theta_{1} \theta_{2}}=\left\{z \in \mathbf{C}: \pi / 2<\theta_{1} \leq|\arg z| \leq \theta_{2}<\pi\right\}
$$

Let the complex function $q(x)$ satisfy the following conditions $q(x) \in C^{1}(\bar{\Omega}, \mathbf{C})$ and

$$
q(x) \in \psi_{\theta_{1} \theta_{2}} .
$$

Then, for sufficiently large in modulus $\lambda$ that

$$
\lambda \in \psi_{\theta_{1} \theta_{2}}
$$

The operator $(P-\lambda I)^{-1}$ exists and is continuous in $H$, and the following estimates are valid, for sufficiently large numbers $M_{\psi}, M^{\prime}{ }_{\psi}$ depending on $\psi_{\theta_{1} \theta_{2}}$ :

$$
\begin{gathered}
\left\|(P-\lambda I)^{-1}\right\| \leq M_{\psi}|\lambda|^{-1}, \\
\left\|\omega^{\alpha} \frac{\partial}{\partial x_{i}}(P-\lambda I)^{-1}\right\| \leq M_{\psi}^{\prime}|\lambda|^{\frac{-1}{2}} .
\end{gathered}
$$

Proof. So, as in Section 1 for a closed extension the operator $P$ (for more explanations, see Ch.6 of [8]), we need to extend its domain to the closed set

$$
D(P)=\left\{u \in \stackrel{\circ}{\mathcal{H}} \cap W_{2, L o c}^{2}(\Omega): P u \in H\right\} .
$$


Let the operator $P$, now satisfy (3.1), (3.2). Then there exist a $c^{\prime}>0$ and complex number $z \in \mathbf{C}$ (noticed that we can take $z=e^{i \gamma}$, for a fix real $\gamma \in$ $(-\pi, \pi])$, such that:

$$
c^{\prime} \leq \operatorname{Re}\{z q(x)\}, \quad c^{\prime}|\lambda| \leq-\operatorname{Re}\{z \lambda\}, \quad\left(\forall x \in \bar{\Omega}, \lambda \in \psi_{\theta_{1} \theta_{2}}\right) .
$$

In view of the uniformly elliptic condition, there is $c>0$ such that:

$$
c|s|^{2}=c \sum_{i=1}^{n}\left|s_{i}\right|^{2} \leq \sum_{i, j=1}^{n} a_{i j}(x) s_{i} \overline{s_{j}}\left(s=\left(s_{1}, \ldots, s_{n}\right) \in \mathbf{C}^{n}, x \in \Omega,\right.
$$

taking $s_{i}=y_{x_{i}}^{\prime}$ implies that

$$
c \sum_{i=1}^{n}\left|y_{x_{i}}^{\prime}(x)\right|^{2} \leq \sum_{i, j=1}^{n} a_{i j}(x) y_{x_{i}}^{\prime}(x) \overline{y_{x_{j}}^{\prime}(x)} .
$$

From this, and according to $c^{\prime} \leq \operatorname{Re}\{z q(x)\}$ in (3.5), we then multiply these two positive relations with each other implies that

$$
c_{1} \sum_{i=1}^{n}\left|y_{x_{i}}^{\prime}(x)\right|^{2} \leq \operatorname{Re}\{z q(x)\} \sum_{i, j=1}^{n} a_{i j}(x) y_{x_{i}}^{\prime}(x) \overline{y_{x_{j}}^{\prime}(x)} .
$$

Multiply both sides of the latter relation by the positive term $\omega^{2 \alpha}(x)$, and then integrate from both sides, we will have

$$
B \leq \operatorname{Re} z \sum_{i, j=1}^{n} \int_{\Omega} \omega^{2 \alpha}(x) a_{i j}(x) q(x) y_{x_{i}}^{\prime}(x) \overline{y_{x_{j}}^{\prime}(x)} d x .
$$

Here $B=c_{1} \sum_{i=1}^{n} \int_{\Omega} \omega^{2 \alpha}(x)\left|y^{\prime}{ }_{x_{i}}(x)\right|^{2} d x$. Now by applying integration by parts, and using Dirichlet-type condition, then the right sides of the latter relation without multiple Re $z$ becomes:

$$
\begin{gathered}
\sum_{i, j=1}^{n} \int_{\Omega} \omega^{2 \alpha}(x) a_{i j}(x) q(x) y_{x_{i}}^{\prime}(x) \overline{y_{x_{j}}^{\prime}(x)} d x \\
=-\sum_{i, j=1}^{n} \int_{\Omega}\left(\omega^{2 \alpha}(x) a_{i j}(x) q(x) y_{x_{i}}^{\prime}(x)\right)_{x_{j}}^{\prime} \bar{y}(x) d x \\
=\left(-\sum_{i, j=1}^{n}\left(\omega^{2 \alpha}(x) a_{i j}(x) q(x) y_{x_{i}}^{\prime}(x)\right)_{x_{j}}^{\prime}, y(x)\right)=(P y, y) .
\end{gathered}
$$


Here, the symbol (,) denotes the inner product in $H$. Notice that the above equality in (3.6) obtains by the well known theorem of the m-sectorial operators which are closed by extending its domain to the closed domain in $\mathcal{H}$. These operators are associated with the closed sectorial bilinear forms that are densely defined in $\mathcal{H}$ (for more explanations, see the well known Theorem 2.1, Chapter 6 of [8]). Therefore,

$$
c_{1} \sum_{i=1}^{n} \int_{\Omega} \omega^{2 \alpha}(x)\left|y^{\prime}{ }_{x_{i}}(x)\right|^{2} d x \leq\{\operatorname{Re} z(P y, y)\}
$$

from (3.5) we have: $c^{\prime}|\lambda| \leq-\operatorname{Re}\{z \lambda\}, c^{\prime}>0, \forall \lambda \in \psi_{\theta_{1} \theta_{2}}$. Multiply this inequality by $\int_{\Omega}|y(x)|^{2} d x=(y, y)=\|y\|^{2}>0$. It follows that: $c^{\prime}|\lambda| \int_{\Omega}|y(x)|^{2} d t \leq$ $-\operatorname{Re}\{z \lambda\}(y, y)$. Now let $K=c_{1} \sum_{i=1}^{n} \int_{\Omega} \omega^{2 \alpha}(x)\left|y^{\prime}{ }_{x_{i}}(x)\right|^{2} d x+c^{\prime}|\lambda| \int_{\Omega}|y(x)|^{2} d x$, then we will have

$$
K \leq \operatorname{Re}\{z(P-\lambda I) y, y)\}
$$

So

$$
K \leq|z|\|y\|\|(P-\lambda I) y\|=\|y\|\|(P-\lambda I) y\|,
$$

i.e.,

$$
c_{1} \sum_{i=1}^{n} \int_{\Omega} \omega^{2 \alpha}(x)\left|y_{x_{i}}^{\prime}(x)\right|^{2} d x+c^{\prime}|\lambda| \int_{\Omega}|y(x)|^{2} d x \leq\|y\|\|(P-\lambda I) y\| .
$$

Since $c_{1} \sum_{i=1}^{n} \int_{\Omega} \omega^{2 \alpha}(x)\left|y_{x_{i}}^{\prime}(x)\right|^{2} d x$ is positive. We will have $c^{\prime}|\lambda|\|y(x)\|^{2}=c^{\prime}|\lambda| \int_{\Omega}|y(x)|^{2} d x \leq\|y\|\|(P-\lambda I) y\|$, or equivalently

$$
|\lambda|\|y(x)\| \leq M_{\psi}\|(P-\lambda I) y\| .
$$

This inequality ensures that the operator $(P-\lambda I)$ is one to one. Therefore the inverse $(P-\lambda I)^{-1}$ exists, and its continuity follows from the proof of the estimate (3.3). To prove (3.3), if we set $y=(P-\lambda I)^{-1} f, f \in H$ in (3.8), then

$$
|\lambda| \int_{\Omega}\left|(P-\lambda I)^{-1} f\right|^{2} d x \leq M_{\psi}\left\|(P-\lambda I)^{-1} f\right\|\left\|(P-\lambda I)(P-\lambda I)^{-1} f\right\| .
$$

Since $(P-\lambda I)(P-\lambda I)^{-1} f=f$, then

$$
|\lambda| \int_{\Omega}\left|(P-\lambda I)^{-1} f\right|^{2} d x \leq M_{\psi}\left\|(P-\lambda I)^{-1} f\right\|\|f\| .
$$

So

$$
|\lambda|\left\|(P-\lambda I)^{-1} f\right\|^{2} \leq M_{\psi}\left\|(P-\lambda I)^{-1} f\right\|\|f\| .
$$


Which this implies that $|\lambda|\left\|(P-\lambda I)^{-1} f\right\| \leq M_{\psi}\|f\|$. Since $\lambda \neq 0$, then

$$
\left\|(P-\lambda I)^{-1}\right\| \leq M_{\psi}|\lambda|^{-1} .
$$

This estimate completes the proof of the assertion (3.3).

Now, we start to prove the estimate (3.4). As in the above argument, we drop the positive term $c^{\prime}|\lambda| \int_{\Omega}|y(x)|^{2} d x$ from

$$
c_{1} \sum_{i=1}^{n} \int_{\Omega} \omega^{2 \alpha}(x)\left|y^{\prime} x_{i}(x)\right|^{2} d x+c^{\prime}|\lambda| \int_{\Omega}|y(x)|^{2} d x \leq\|y\|\|(P-\lambda I) y\| .
$$

It follows that

$$
c_{1} \sum_{i=1}^{n} \int_{\Omega} \omega^{2 \alpha}(x)\left|y_{x_{i}}^{\prime}(x)\right|^{2} d x \leq\|y\|\|(P-\lambda I) y\| .
$$

Then,

$$
c_{1}\left\|\omega^{\alpha} \frac{\partial}{\partial x_{i}}(P-\lambda I)^{-1} f\right\|^{2} \leq\|y\|\|(P-\lambda I) y\| .
$$

Set $y=(P-\lambda I)^{-1} f, f \in H$ in the latter relation, and proceeding by similar calculation as in the proof (3.3), we then obtain:

$$
c_{1}\left\|\omega^{\alpha} \frac{\partial}{\partial x_{i}}(P-\lambda I)^{-1} f\right\|^{2} \leq\left\|(P-\lambda I)^{-1} f\right\|\left\|(P-\lambda I)(P-\lambda I)^{-1} f\right\| .
$$

Since $(P-\lambda I)(P-\lambda I)^{-1} f=I(f)=f$, then

$$
c_{1}\left\|\omega^{\alpha} \frac{\partial}{\partial x_{i}}(P-\lambda I)^{-1} f\right\|^{2} \leq\left\|(P-\lambda I)^{-1}\right\| f \|^{2},
$$

consequently, by (3.3) this implies that

$$
c_{1}\left\|\omega^{\alpha} \frac{\partial}{\partial x_{i}}(P-\lambda I)^{-1} f\right\|^{2} \leq M_{\psi}|\lambda|^{-1}\|f\|^{2},
$$

to this end we will have

$$
\left\|\omega^{\alpha} \frac{\partial}{\partial x_{i}}(A-\lambda I)^{-1}\right\| \leq M_{\psi}^{\prime}|\lambda|^{-\frac{1}{2}} .
$$

Thus, here the proof of the estimate (3.4) is finished; i.e., this completes the proof of Theorem 4. 


\section{On the resolvent estimate of the differential operator in $H_{\ell}$}

As in Section 1, let the differential operator

$$
(P u)(x)=-\sum_{i, j=1}^{n}\left(\omega^{2 \alpha}(x) a_{i j}(x) Q(x) u_{x_{i}}^{\prime}(x)\right)_{x_{j}}^{\prime}
$$

act in Hilbert space $H_{\ell}=L^{2}(\Omega)^{\ell}$ with Dirichlet-type boundary conditions, and suppose that $Q(x) \in C^{2}\left(\bar{\Omega}\right.$, End $\left.\mathbf{C}^{\ell}\right)$ such that for each $x \in \bar{\Omega}$ the matrix function $Q(x)$ has non-zero simple eigenvalues $\mu_{j}(x) \in C^{2}(\bar{\Omega}) \quad(1 \leq j \leq \ell)$ arranged in the complex plane in the following way:

$$
\mu_{1}(x), \ldots, \mu_{\nu}(x) \in \mathcal{R}^{+}, \mu_{\nu+1}(x), \ldots, \mu_{\ell}(x) \in \psi_{\theta_{1} \theta_{2}}
$$

where

$$
\psi_{\theta_{1} \theta_{2}}=\left\{z \in \mathbf{C}: \pi / 2<\theta_{1} \leq|\arg z| \leq \theta_{2}<\pi\right\} .
$$

Theorem 5. Let (4.1) and the assumptions of Section 1 hold for the operator $P$, then for sufficiently large in modulus $\lambda \in \psi_{\theta_{1} \theta_{2}}$, the inverse $(P-\lambda I)^{-1}$ exists and is continuous in the space $H_{\ell}=L^{2}(\Omega)^{\ell}$ and the following estimate holds:

$$
\left\|(P-\lambda I)^{-1}\right\| \leq M_{\psi}|\lambda|^{-1},
$$

where $M_{\psi}>0$ is sufficiently large number depending on $\psi_{\theta_{1} \theta_{2}}$ and $|\lambda|>C_{\psi_{\theta_{1} \theta_{2}}}$ and $C_{\psi_{\theta_{1} \theta_{2}}}>0$ is a constant number.

Proof. Now by applying the eigenvalues $\mu_{1}(x), \ldots, \mu_{\ell}(x)$ of the matrix function $Q(x)$ we defined the operators $P_{1}, \ldots, P_{\ell}$ such that:

$$
\left(P_{k} u\right)(x)=-\sum_{i, j=1}^{n}\left(\omega^{2 \alpha}(x) a_{i j}(x) \mu_{k}(x) u_{x_{i}}^{\prime}(x)\right)_{x_{j}}^{\prime}, \quad(k=1, \ldots, \ell),
$$

where its extension domains are:

$$
D\left(P_{k}\right)=\left\{u \in \stackrel{\circ}{\mathcal{H}} \bigcap W_{2, L o c}^{2}(\Omega): P_{k} u \in H\right\},
$$

which as the operator $P$ in Theorem 4 , the operators $P_{k}, k=1, \ldots, \ell$, act in space $H=L^{2}(\Omega)$ (Notice that here the operators $P_{k}$ are the same of the operator $P$ in Section 2, i.e., to define the operators $P_{k}$, we just change the function $q(x)$ in the operator $P$ by the eigenvalues functions $\mu_{k}(x), k=1, \ldots, \ell$ of matrix $Q(x)$ ). 
The conditions which we consider on the eigenvalues $\mu_{j}(x)$ of the matrix function $Q(x)$ in Section 1 guarantee that one can convert the matrix $Q(x)$ to the diagonal form

$$
Q(x)=U(x) \Lambda(x) U^{-1}(x),
$$

where $U(x), U^{-1}(x) \in C^{2}\left(\bar{\Omega}\right.$, End $\left.\mathbf{C}^{\ell}\right)$ and

$$
\Lambda(x)=\operatorname{diag}\left\{\mu_{1}(x), \ldots, \mu_{\ell}(x)\right\} .
$$

Consider space $H_{\ell}=H \oplus \cdots \oplus H \quad(\ell$-times $)$ and put $\Gamma(\lambda)=U B(\lambda) U^{-1}$, where the operator

$$
B(\lambda)=\operatorname{diag}\left\{\left(P_{1}-\lambda I\right)^{-1}, \ldots,\left(P_{\ell}-\lambda I\right)^{-1}\right\},
$$

act in the direct sum

$$
H_{\ell}=H \oplus \cdots \oplus H \quad(\ell \text {-times }) .
$$

The following equation has been proved in the [10],[11]

$$
A=I+\omega^{2 \alpha-1}(x) q_{0}(x) B(\lambda) U^{-1}+\omega^{2 \alpha}(x) \sum_{i=1}^{n}\left(q_{i}(x) \frac{\partial}{\partial x_{i}} B(\lambda) U^{-1}\right) .
$$

Here $A=(P-\lambda I) \Gamma(\lambda)$ and $q_{i} \in C\left(\bar{\Omega}\right.$, End $\left.\mathbf{C}^{\ell}\right), i=0,1,2, \ldots, n$. Now let us take the right side of (4.3) equals to $I+T^{\prime}(\lambda)$. Thus, we will have

$$
(P-\lambda I) \Gamma(\lambda)=I+T^{\prime}(\lambda) .
$$

Now according to Section 3 if we put $P_{k}=P$ for $k=1, \ldots, \ell$ in (3.3) we will have

$$
\left\|\left(P_{k}-\lambda I\right)^{-1}\right\| \leq M_{1 \psi_{\theta_{1} \theta_{2}}}|\lambda|^{-1},
$$

where $\lambda \in \psi_{\theta_{1} \theta_{2}},|\lambda|>C_{\psi_{\theta_{1} \theta_{2}}}$ and $M_{1 \psi_{\theta_{1} \theta_{2}}}, C_{\psi_{\theta_{1} \theta_{2}}}>0$ are sufficiently large numbers depending on $\psi_{\theta_{1} \theta_{2}}$. Owing to the latter relations and the definition of $T^{\prime}(\lambda)$ in (4.4) and Hardy inequality we will have

$$
\left\|T^{\prime}(\lambda)\right\| \leq M_{\psi_{\theta_{1} \theta_{2}}}|\lambda|^{-\frac{1}{2}},\left(\lambda \in \psi_{\theta_{1} \theta_{1}}, \quad|\lambda|>1\right) .
$$

Here $M_{\psi_{\theta_{1} \theta_{2}}}>0$ is a sufficiently large number. Since $|\lambda|$ is sufficiently large number, easily implies that $\left\|T^{\prime}(\lambda)\right\|<\frac{1}{2}<1$, from this and using the well known theorem in the operator theory we conclude that $I+T^{\prime}(\lambda)$ and so $(P-\lambda I) \Gamma(\lambda)$ are invertible. Hence, $((P-\lambda I) \Gamma(\lambda))^{-1}$ exists and equals to

$$
(\Gamma(\lambda))^{-1}(P-\lambda I)^{-1}=\left(I+T^{\prime}(\lambda)\right)^{-1} .
$$


By adding $+I$ and $-I$ to the right side of (4.6), it follows that

$$
(\Gamma(\lambda))^{-1}(P-\lambda I)^{-1}=\left(I+T^{\prime}(\lambda)\right)^{-1}-I+I
$$

We now set

$$
F^{\prime}(\lambda)=\left(I+T^{\prime}(\lambda)\right)^{-1}-I
$$

Then

$$
(\Gamma(\lambda))^{-1}(P-\lambda I)^{-1}=I+F^{\prime}(\lambda) .
$$

In view of $\left\|T^{\prime}(\lambda)\right\|<1$ and (4.6), we now estimate $F^{\prime}(\lambda)$ by the following geometric series:

$$
\begin{aligned}
& \left\|F^{\prime}(\lambda)\right\| \leq \sum_{k=2}^{+\infty}\left\|T^{\prime k}(\lambda)\right\| \leq\left\|T^{\prime}(\lambda)\right\|^{2}\left(1+\left\|T^{\prime}(\lambda)\right\|+\left\|T^{\prime}(\lambda)\right\|^{2}+\ldots\right) \\
& \leq\left\|T^{\prime}(\lambda)\right\|^{2}(1+1 / 2+\ldots) \leq 2 M_{\psi_{\theta_{1} \theta_{2}}|\lambda|^{-1}}^{2}
\end{aligned}
$$

i.e., $\left\|F^{\prime}(\lambda)\right\| \leq 2 M_{\psi_{\theta_{1} \theta_{2}}}^{2}|\lambda|^{-1}$. By $\left\|\left(P_{k}-\lambda I\right)^{-1}\right\| \leq M_{1 \psi_{\theta_{1} \theta_{2}}}|\lambda|^{-1}$ in view of definition $B(\lambda)$ and $\Gamma(\lambda)$ we will have

$$
\|\left(\Gamma(\lambda) \| \leq M_{2 \psi_{\theta_{1} \theta_{2}}}|\lambda|^{-1}\right.
$$

Now from (4.6) we have

$$
\left\|(P-\lambda I)^{-1}\right\| \leq \|\left(\Gamma ( \lambda ) \| \| ( I + T ^ { \prime } ( \lambda ) ) ^ { - 1 } \| = \| \left(\Gamma(\lambda)\|\|\left(I+F^{\prime}(\lambda)\right) \| .\right.\right.
$$

Therefore,

$$
\left\|(P-\lambda I)^{-1}\right\| \leq\|\Gamma(\lambda)\|\left\|\left(I+F^{\prime}(\lambda)\right)\right\|
$$

So,

$$
\left\|(P-\lambda I)^{-1}\right\| \leq M_{2 \psi_{\theta_{1} \theta_{2}}}|\lambda|^{-1}\left(1+2 M_{\psi_{\theta_{1} \theta_{2}}}^{2}|\lambda|^{-1}\right) .
$$

Now $M_{2 \psi_{\theta_{1} \theta_{2}}}|\lambda|^{-1}\left(1+2 M_{\psi_{\theta_{1} \theta_{2}}}^{2}|\lambda|^{-1}\right) \leq M_{2 \psi_{\theta_{1} \theta_{2}}}|\lambda|^{-1}\left(1+2 M_{\psi_{\theta_{1} \theta_{2}}}^{2}\right)$. So,

$$
\left\|(P-\lambda I)^{-1}\right\| \leq M_{\psi}|\lambda|^{-1}
$$

where $M_{\psi}|\lambda|^{-1}=M_{2 \psi_{\theta_{1} \theta_{2}}}|\lambda|^{-1}\left(1+2 M_{\psi_{\theta_{1} \theta_{2}}^{2}}\right)$. 


\section{References}

[1] M.S. Agranovich, Elliptic operators on closed manifolds, Partial Differential Equations-6, Itogi Nauki i Tekhniki. Ser. Sovrem. Probl. Mat. Fund. Napr., 63, VINITI, Moscow (1990), 5-129 (In Russian).

[2] K.Kh. Boimatov, A.G. Kostyuchenko, Distribution of eigenvalues of second-order non-selfadjoint differential operators, Vest. Mosk. Gos. Univ., Ser. I, Mat. Mekh., 45, No 3 (1990), 24-31 (In Russian).

[3] K.Kh. Boimatov, Asymptotic behaviour of the spectra of second-order nonselfadjoint systems of differential operators, Mat. Zametki, 51, No 4 (1992), 6-16 (In Russian).

[4] K.Kh. Boimatov, Asymptotics of the spectrum of an elliptic differential operator in the degenerate case, Dokl. Akad. Nauk SSSR, 243, No 6 (1978), 1369-1372 (In Russian).

[5] K.Kh. Boimatov, Separability theorems, weighted spaces and their applications, In: Investigations in the Theory of Differentiable Functions of Many Variables and its Applications. Part 10, Collection of Articles, Trudy Mat. Inst. Steklov, 170 (1984), 37-76; Proc. Steklov Inst. Math., 170 (1987), 39-81 (In Russian).

[6] K.Kh. Boimatov, Spectral asymptotics of pseudodifferential operators, Dokl. Akad. Nauk SSSR, 311, No 1 (1990), 14-18; Dokl. Math., 41, No 2 (1990), 196-200 (In Russian).

[7] I.C. Gokhberg, M.G. Krein, Introduction to the Theory of Linear Nonselfadjoint Operators, American Mathematical Society, Providence (1969).

[8] T. Kato, Perturbation Theory for Linear Operators, Springer, New York (1966).

[9] M.A. Naymark, Linear Differential Operators, Moscow, Nauka Publ., (1969) (In Russian).

[10] A. Sameripour, K. Seddighi, Distribution of eigenvalues of nonself-adjoint elliptic systems degenerate on the domain boundary, Mat. Zametki, 61, No 3 (1997), 463-467; Math. Notes, 61, No 3 (1997), 379-384; doi: https://doi.org/10.4213/mzm1524 (In Russian). 
[11] A. Sameripour, K. Seddighi, On the spectral properties of generalized non-selfadjoint elliptic systems of differential operators degenerated on the boundary of domain, Bull. Iranian Math. Soc., 24, No 1 (1998), 15-32.

[12] A.A. Shkalikov, Theorems of Tauberian type on the distribution of zeros of holomorphic functions, Mat. Sb. (N.S.), 123 (165), No 3 (1984), 317-347; Math. USSR-Sb., 51, No 2 (1985) 315-344 (In Russian). 